\title{
Tradução e adaptação cultural do questionário PRTEE (Patient-rated Tennis Elbow Evaluation) para a língua portuguesa ${ }^{1}$
}

\author{
Caroline Sousa de Andrade, Regyane Costa Souza, Therezinha Rosane Chamlian, \\ Marcelo Hide Matsumoto, João Baptista Gomes dos Santos, Akira Ishida
}

Resumo: Introdução: Frequentemente encontrada em clínicas de reabilitação, a epicondilite lateral pode causar dor e diminuição funcional. Sua avaliação é considerada de fundamental importância; entretanto, algumas podem carecer de maiores informações funcionais do indivíduo. No Brasil, essa desordem não possui instrumento específico de avaliação. O desenvolvimento do PRTEE (Patient-rated Tennis Elbow Evaluation), no Canadá, possibilita sua mensuração, por meio de uma abordagem sistemática de tradução e adaptação cultural do questionário. Objetivos: Traduzir a versão da escala PRTEE da língua inglesa para a portuguesa; verificar o nível de compreensão entre os profissionais da saúde e as pessoas leigas; identificar sugestões quanto à estrutura, e desenvolver uma nova versão adaptada culturalmente para a população brasileira. Métodos: Foram realizadas duas traduções da escala original inglesa para o português, as quais foram retrotraduzidas em duas versões no inglês. Comparadas à versão original, estas foram analisadas e, na presença de divergências, modificadas. Uma versão brasileira do PRTEE foi obtida e avaliada em relação à sua equivalência cultural e ao conteúdo. Para verificar seu nível de compreensão, a medida foi aplicada com profissionais da saúde (48) e leigos (52). No processo de equivalência cultural do instrumento, as questões com o índice de 'não compreensão' foram reformuladas. Resultados: Em primeira aplicação, três itens do questionário atingiram porcentagem superior a 15\% de 'não compreensão'. Substituídos e reaplicados, esses itens apresentaram alta porcentagem de compreensão. Conclusão: Construiu-se uma versão adaptada culturalmente do instrumento aos profissionais da saúde e pessoas leigas da população brasileira.

Palavras-chave: Escalas, Estudos de Validação, Cotovelo de Tenista, Avaliação.

\section{Translation and cross-cultural adaptation of the PRTEE (Patient-rated Tennis Elbow Evaluation) questionnaire to Portuguese}

\begin{abstract}
Introduction: Frequently found in clinical rehabilitation, tennis elbow may induce pain and functional decline. Its evaluation is considered crucial; however, some evaluations lack more individuals' functional information. In Brazil, this disorder doesn't have any specific evaluation tools. The development of the 'Patient-rated Tennis Elbow Evaluation' (PRTEE) questionnaire, in Canada, has enabled tennis elbow measurement through a systematic approach of translation and cultural adaptation of the survey. Purpose: To translate the English version of the Patient-rated Tennis Elbow Evaluation questionnaire to Portuguese; check the level of understanding among health professionals and laymen; identify suggestions regarding its structure; and develop a new version, culturally adapted to the Brazilian population. Methods: Two translations of the original English version to Portuguese were prepared; they were then translated back in two new English versions. The two new English translations were compared to the original English version; they were analyzed and, in the presence of differences, modified. A Brazilian version of the Patient-rated Tennis Elbow Evaluation was obtained and assessed concerningits contents and cultural equivalence. In order to verify the level of understanding, the questionnaire was applied to health professionals (48) and laymen (52). In the process of cultural equivalence of the instrument, the questions indexed as not-understood were reformulated. Results: In the first application, three items of the questionnaire reached a percentage greater than $15 \%$ of not-understood. After being reformulated and reapplied, these items showed high percentage of understanding. Conclusion: A culturally adapted version of the Patient-rated Tennis Elbow Evaluation (PRTEE) was developed to Brazilian health professionals and laymen.
\end{abstract}

Keywords: Scales, Validation Studies, Tennis Elbow, Evaluation.

Autor para correspondência: Caroline Sousa de Andrade, Universidade Potiguar - UnP, Av. Senador Salgado Filho, Lagoa Nova, CEP 59056-000, Natal, RN, Brasil, e-mail: tocarolinesousa@yahoo.com.br

Recebido em 23/9/2010; $1^{\text {a }}$ Revisão em 6/5/2011; $2^{\text {a }}$ Revisão em 30/8/2011; Aceito em 7/11/2011 


\section{Introdução}

Segundo Freitas (2006), a epicondilite lateral é considerada uma das mais frequentes desordens do cotovelo encontradas em clínicas de reabilitaçáo do membro superior. Essa doença engloba uma tendinite que ocorre no local de origem dos tendóes extensores, na extremidade distal do úmero, com etiologia relacionada a traumas, estiramentos (agudos ou crônicos) e atividades ocupacionais ou esportivas (PARDINI JUNIOR, 2000).

O paciente com epicondilite lateral relata dor na região do epicôndilo lateral (FREITAS, 2006; LECH; SEVERO, 2003; PARDINI JUNIOR; FREITAS; TAVARES, 2003), que se irradia ao longo da musculatura extensora. Pode haver diminuição da capacidade funcional do indivíduo, resultando na não realização de atividades diárias comuns $(\mathrm{LECH}$; SEVERO, 2003). Em alguns casos, é possível haver referência à dor no final do dia e sensaçáo de rigidez ao acordar (FREITAS, 2006), bem como limitaçáo de movimento e edema (FREITAS, 2006; PARDINI JUNIOR; FREITAS; TAVARES, 2003).

Embora com tratamento inicial conservador (FREITAS, 2006; LECH; SEVERO, 2003; PARDINI JUNIOR; FREITAS; TAVARES, 2003), o tratamento cirúrgico também é realizado após 6 a 8 meses de evolução ou quando ocorrerem várias recidivas, que dificultam as atividades profissionais e de lazer do indivíduo (LECH; SEVERO, 2003).

O terapeuta deve sempre realizar avaliação do paciente antes de tratá-lo. O conhecimento da história da doença, a realização de exame físico e o grau de comprometimento funcional podem fornecer dados relevantes para o tratamento (FREITAS, 2006). Contudo, Freire, Souza e Lima (2007) relatam que as informaçóes obtidas por meio de avaliação física nem sempre fornecem dados a respeito da funcionalidade do cotovelo ou o quanto as atividades de vida diária (AVD's) do paciente podem estar comprometidas.

A interpretaçáo dos resultados referentes ao tratamento da epicondilite lateral torna-se de difícil acesso por causa da ausência de consenso de medidas padronizadas (NILSSON et al., 2008; ROMPE; OVEREND; MACDERMID, 2007). Alguns questionários, segundo esses últimos autores, têm sido utilizados para avaliar essa doença, tais como Dash (Disabilities of arm, shoulder and hand) e UEFS (Upper extremity function scale). Entretanto, esses questionários gerais, para os autores, podem não avaliar com precisão os sintomas e a funçáo de uma articulação. Desenvolvido para a epicondilite lateral, o "The Roles and Maudsley Outcome Scores" é uma avaliação que sugere caracterização genérica do tratamento (ALTAN et al., 2010) como excelente, bom, regular e pobre (ALTAN et al., 2010; LEUNG; YEN; TSE, 2004).

Paralelamente ao desenvolvimento de sistemas de classificação, um questionário foi desenvolvido por MacDermid e colaboradores, direcionado exclusivamente a pacientes com epicondilite lateral, o Patient-rated Tennis Elbow Evaluation - PRTEE (ROMPE; OVEREND; MACDERMID, 2007). Inicialmente conhecido como Patient-rated Forearm Evaluation Questionnaire - PRFEQ (MACDERMID, 2007), esse questionário foi desenvolvido no ano de 1999 no Canadá (NILSSON et al., 2008). Em 2005, essa escala até então denominada PRFEQ obteve ligeiras atualizaçóes por seu idealizador, MacDermid, e passou a se chamar PRTEE (MACDERMID, 2005).

Em 2007, a versão atualizada, PRTEE, foi validada por Rompe, Overend e MacDermid (2007), em uma amostra de pacientes jogadores de tênis, sendo considerada um instrumento confiável, reproduzível e sensível para avaliar a epicondilite lateral nessa população. Nesse mesmo estudo, foi relatado que o PRTEE pode tornar-se padrão primário de medida em pesquisas dessa doença (ROMPE; OVEREND; MACDERMID, 2007).

O "Patient-rated Tennis Elbow Evaluation" é um questionário formado por 15 itens (MACDERMID, 2007), os quais avaliam média de dor e função em pacientes com epicondilite lateral no braço afetado durante a semana que antecede sua aplicação. Esse espaço de tempo permite a recordação exata da resposta ao mesmo tempo em que evita efeitos de flutuação aguda nos sintomas.

O PRTEE consiste de duas subescalas (ROMPE; OVEREND; MACDERMID, 2007). A primeira, com 5 questóes, avalia o domínio dor e a segunda, com 10 questóes, refere-se à função (NILSSON et al., 2008; ROMPE; OVEREND; MACDERMID, 2007). No que se refere à pontuação destas, segundo o último autor, a primeira subescala é pontuada variando de 0 (sem dor) a 10 (pior dor imaginável) e a segunda subescala, de 0 (nenhuma dificuldade) a 10 (incapaz de realizar).

A pontuação final do questionário é obtida por meio da pontuação total da subescala de dor (de 50 pontos) somada à subescala de função (60 pontos para atividades específicas mais 40 de atividades usuais que são então divididos por dois) (MACDERMID, 2007; ROMPE; OVEREND; MACDERMID, 2007). Essa soma fornece uma pontuaçáo total final que varia entre 0 (sem dor e nenhum prejuízo funcional) a 100 
(pior dor imaginável e importante déficit funcional) (ROMPE; OVEREND; MACDERMID, 2007).

Quando ainda chamada PRFEQ, a medida de avaliação obteve sua versão chinesa (LEUNG; YEN; TSE, 2004); depois de considerada PRTEE, por meio da metodologia proposta por Beaton et al. (2000), obtiveram-se suas versôes sueca (NILSSON et al., 2008) e turca (ALTAN et al., 2010).

A utilização de medidas de avaliação tem sido cada vez mais recorrente em ensaios clínicos e pesquisas de saúde pública; assim, com poucas exceçôes, todas as medidas desenvolvidas até agora se encontram em língua inglesa. Embora tal predominância seja observada, é enfatizada a necessidade - que se tornou mais aguda com o crescente número de trabalhos multicêntricos entre vários países - de medidas especificamente projetadas para serem usadas em países com outras línguas e também com população imigrante.

No intuito de atender tal necessidade, duas opçôes são consideradas válidas: 1) o desenvolvimento de uma nova medida de avaliação ou 2) a utilização de uma medida previamente desenvolvida em outra língua. A realização da segunda opção, para sua utilização, requer o desenvolvimento de uma abordagem sistemática de tradução e processo de adaptação cultural (GUILLEMIN; BOMBARDIER; BEATON, 1993).

A frequente produção de instrumentos adaptados dentro de cada país pode representar um grande problema, quando várias versōes são produzidas nas mesmas configuraçôes culturais por equipes independentes. Nesse sentido, menciona-se que cada estudo deve utilizar uma versão referenciada com documentado método de adaptação e validade, no intuito de rejeitar o uso de tradução "bruta", sem um cuidadoso processo de adaptação e validação (GUILLEMIN, 1995).

O processo de tradução e adaptação cultural é baseado em cinco critérios: 1) tradução; 2) retrotradução; 3) revisão do comitê; 4) pré-teste, e 5) ponderação de pontuaçóes.

$\mathrm{Na}$ fase de tradução, se produzem várias traduçôes, com tradutores qualificados; na etapa de retrotradução, realiza-se o maior número de traduçóes na língua de origem como as realizadas para a língua alvo. Sua realização é feita por apropriados retrotradutores; para a etapa de revisão do comitê, constitui-se uma comissão a fim de produzir uma versão final da medida modificada, baseada nas várias traduçốes e retrotraduçôes. A comissão, a qual deve ser multidisciplinar, tem papel de assegurar que a traduçáo seja totalmente compreensível.
$\mathrm{Na}$ presença de itens irrelevantes, inadequados e ambíguos, o comitê pode modificá-los ou eliminá-los, gerando substitutos melhor adaptados à situação da cultura alvo enquanto mantém o conceito geral dos itens excluídos. O comitê verifica equivalência transcultural da versão fonte (origem) e da final (língua alvo), o que envolve equivalência semântica, idiomática, experiencial e conceitual; na quarta fase, realiza-se pré-teste da versão final (na língua alvo) para se verificar equivalência com a versão origem. $\mathrm{Na}$ aplicação deste, uma amostra da população responde ao questionário a fim de checar erros e desvios na tradução. Duas técnicas são válidas para tal: uma técnica de teste ou uma técnica com avaliação por indivíduos bilíngues; e, na última etapa, considera-se a adaptaçâo do peso das pontuaçóes para o contexto cultural (GUILLEMIN; BOMBARDIER; BEATON, 1993).

A ausência de medidas padronizadas para avaliaçáo do tratamento da epicondilite lateral (NEWCOMER et al., 2005; NILSSON et al., 2008; ROMPE; OVEREND; MACDERMID, 2007) e o desenvolvimento de um questionário exclusivo para pacientes com epicondilite lateral (ROMPE; OVEREND; MACDERMID, 2007) são fatores que justificaram a realizaçáo do processo de adaptação cultural do PRTEE no Brasil.

Este estudo tem como objetivo traduzir a versão da escala Patient-rated Tennis Elbow Evaluation (PRTEE) da língua inglesa para a portuguesa, além de verificar o nível de compreensão entre os profissionais da saúde e as pessoas leigas, identificar sugestóes quanto à estrutura e desenvolver uma nova versão adaptada culturalmente para a população brasileira.

\section{Material e métodos}

Selecionaram-se, no Lar Escola São Francisco - Centro de Reabilitação (LESF), setor de Terapia Ocupacional e serviço de Terapia da Mão e Membro Superior, e no Hospital São Paulo (HSP), Ambulatório da Mão e Ambulatório do Ombro e Cotovelo, ambos vinculados à Universidade Federal de São Paulo - Escola Paulista de Medicina (UNIFESP/ EPM), 140 participantes, que foram divididos em duas amostras: uma primeira com 100 participantes e a segunda, com 40. Dentre os participantes, figuraram funcionários e pessoas externas aos locais de seleção, de ambos os gêneros, com faixa etária entre 18 e 59 anos, alfabetizados (Ensino Fundamental, Médio ou Superior), profissionais da área da saúde (fisioterapeutas, terapeutas ocupacionais, enfermeiros, técnicos/auxiliares de enfermagem, nutricionistas e 
médicos) ou pessoas leigas a essa área, que foram divididos em dois grupos: leigos e profissionais.

Indivíduos com déficit cognitivo de qualquer etiologia e lesôes no sistema musculoesquelético do membro superior foram excluídos da amostra.

A primeira amostra se constituiu por 100 participantes, que foram subdivididos em dois grupos: o grupo de profissionais da saúde, composto por 48 participantes, e o grupo de leigos, constituído de 52 participantes. O primeiro grupo formou-se, em sua maioria, por indivíduos do gênero feminino (81,25\%), sobre o gênero masculino (18,75\%); apresentou idade variante entre 22 e 58 anos, e formação diversificada, com terapeutas ocupacionais $(35,42 \%)$, fisioterapeutas (31,25\%), médicos $(14,58 \%)$, auxiliar/técnico de enfermagem $(10,42 \%)$, nutricionistas $(6,25 \%)$ e enfermeira (2,08\%).

O segundo grupo se constituiu, em sua maioria, por indivíduos do gênero feminino (59,62\%), sobre o gênero masculino (41,38\%); apresentou idade variante entre 19 e 59 anos, e nível de escolaridade predominante do Ensino Médio (53,85\%), sobre o Ensino Fundamental $(32,69)$ e o Superior $(13,46 \%)$.

A segunda amostra, adicional, constituiu-se por 40 participantes, que foram divididos em dois grupos, de profissionais da saúde e de leigos, sendo cada qual formado por 20 participantes.

O grupo de profissionais da saúde foi composto predominantemente por mulheres $(80 \%)$, sobre homens (20\%); apresentou idade variante entre 23 e 45 anos, e formação diversificada de terapeutas ocupacionais (40\%), médicos (20\%), fisioterapeutas (20\%), enfermeiro/auxiliar de enfermagem (10\%) e nutricionistas (10\%). O grupo de leigos constituiu-se em sua maioria por mulheres (55\%), sobre homens (45\%), apresentou idade variante entre 19 e 57 anos, e nível de escolaridade predominante do Ensino Médio (50\%), sobre o Ensino Fundamental (25\%) e o Superior (25\%).

Aprovado pelo Comitê de Ética em Pesquisa da Universidade Federal de São Paulo, o trabalho foi iniciado com a solicitação de consentimento ao autor do questionário PRTEE - MacDermid, para realização do processo de tradução e adaptação cultural do instrumento para a língua portuguesa.

Com a autorização obtida e conforme os critérios propostos por Guillemin, Bombardier e Beaton (1993), a versão original em inglês do questionário PRTEE foi encaminhada a dois tradutores juramentados independentes, que traduziram o instrumento para a língua portuguesa, sua língua mãe, e geraram duas versôes do questionário. Nesse momento, apenas um dos dois tradutores estava ciente dos objetivos do estudo.

Em fase seguinte, as duas versôes do questionário em português foram retrotraduzidas para seu idioma de origem (inglês), de forma independente, por outros dois tradutores, também juramentados. Os retrotradutores náo estavam cientes da intenção e dos conceitos subjacentes ao material. Nessa etapa, equívocos na primeira traduçáo, falhas para adaptar para o contexto cultural alvo e ambiguidades não foram encontrados.

Para prosseguir, de acordo com as etapas propostas, constituiu-se um comitê, que foi formado por uma tradutora juramentada e uma terapeuta ocupacional, com conhecimentos da língua inglesa e dos conceitos envolvidos no trabalho. Essa comissão analisou o material recolhido e selecionou as perguntas/ termos mais elaborados, de fácil compreensão de cada versão, que integraram uma única versão na língua portuguesa.

Para verificação de equivalência entre a medida original (inglês) e essa versão final (na língua portuguesa), inicialmente explicou-se aos 100 participantes o objetivo do estudo e solicitou-se a estes que assinassem o termo de consentimento. Uma ficha de identificação também foi entregue aos mesmos. A versão final em português, acrescida dos termos 'compreensível' e 'não compreensível', além de um espaço destinado à colocação dos itens que não estavam claros ou dos termos que deveriam ser modificados, foi aplicada aos participantes.

Durante o preenchimento desse questionário, os participantes foram encorajados a elucidar sua compreensão a respeito de cada pergunta e assinalar, para cada item, se o mesmo estava compreensível ou não para a língua portuguesa.

Mensurou-se a porcentagem dos níveis de compreensão e não compreensão dos itens do questionário, realizaram-se mudanças, reaplicou-se o teste e mensurou-se novamente o nível de compreensão e não compreensão dos itens.

\section{Limitações do estudo}

As amostras, que foram subdivididas em grupos de profissionais e leigos, tiveram este último constituído, na grande maioria, por indivíduos com escolaridade Média e Fundamental, o que não representa a realidade da população brasileira que busca os serviços 
de saúde pública. Esse fator pode ter influenciado os resultados da pesquisa.

\section{Resultados}

Obteve-se, com a aplicação da medida final em português, na amostra composta por 100 participantes, nível superior a $15 \%$ de náo compreensão para três itens: "Quando sua dor foi a menor", com 47\% de não compreensão; "Quando sua dor foi a pior", com 46\%, e "Atividades recreativas ou de esportes" com 18\%, como pode ser visto na Tabela 1. Por ter se embasado no estudo de Guillemin (2007 apud FREIRE;SOUZA;LIMA, 2007), que propóe adotar nível de não compreensão de $15 \%$, esses itens que atingiram porcentagem superior à estabelecida foram modificados, de acordo com sugestóes dos participantes envolvidos e da comissão, como pode ser visto no Quadro 1; esses itens foram reaplicados por meio de nova versão final em participantes que constituíram uma nova amostra (adicional), que foi composta por 40 participantes.

Um alto grau de compreensão dos itens foi obtido, o que representou sua equivalência à versão original. A Tabela 2 apresenta o nível de compreensão obtido para cada questão.

\section{Discussão}

Embora considerada uma das mais frequentes desordens do cotovelo (FREITAS, 2006), a epicondilite não possui instrumento específico de avaliação no Brasil. Para Nilsson et al. (2008), uma avaliação baseada no PRTEE poderia facilitar a comparação dos efeitos de tratamento e da tomada de decisóes sobre a melhor forma para tratar a epicondilite lateral.

Em seu trabalho, Newcomer et al. (2005) relatam que, nos estudos prévios referentes à epicondilite lateral, pesquisadores realizavam mensuração dos resultados do tratamento por meio da combinação de força de preensão e sensibilidade à dor/ pontuação de dor, utilizando escalas não padronizadas. Outras medidas de resultado são citadas por esses autores como comumente utilizadas: o Dash (Disabilities of arm, shoulder and hand), VAS (Visual analogue scale) e PFG (Pain-free grip) medida de força. O SF-36 também é referido, avaliando o estado geral de saúde do paciente.

Rompe, Overend e Macdermid (2007), além do Dash, fazem referência ao UEFS (Upper extremity function scale) como medida de avaliação também utilizada para epicondilite lateral. Entretanto, os mesmos descrevem que questionários gerais podem não avaliar com precisão os sintomas e a função de uma articulação. Desenvolvido especificamente para avaliar a epicondilite lateral, o "The Roles and Maudsley Outcome score" é citado por Altan et al. (2010) como uma avaliação que apresenta genérica pontuação e que carece de informaçôes detalhadas sobre a avaliação funcional. Para Leung, Yen e Tse (2004), a pontuação desse questionário restringe a

Tabela 1. Porcentagem de compreensão dos itens da medida final em português.

\begin{tabular}{lcc}
\hline \multicolumn{1}{c}{ Questão } & $\begin{array}{c}\text { Compreensível } \\
(\mathbf{\%})\end{array}$ & $\begin{array}{c}\text { Não compreensível } \\
\text { (\%) }\end{array}$ \\
\hline Quando você está descansando & 97 & 3 \\
Quando você está realizando uma tarefa com movimentos & 95 & 5 \\
repetitivos do braço & & \\
Quando você está carregando uma sacola plástica com & 99 & 1 \\
artigos de supermercado & 53 & 47 \\
Quando sua dor foi a menor & 54 & 46 \\
Quando sua dor foi a pior & 96 & 4 \\
Girar uma maçaneta ou chave & 99 & 1 \\
Carregar uma sacola de supermercado ou pasta pelas alças & 100 & 0 \\
Levantar uma xícara de café ou copo de leite até sua boca & 100 & 0 \\
Abrir a tampa de um pote & 100 & 0 \\
Vestir calças & 100 & 0 \\
Torcer um pano de limpeza ou uma toalha molhada & 94 & 6 \\
Atividades pessoais (vestir-se, tomar banho) & 98 & 2 \\
Trabalhos domésticos (limpeza, manutenção) & 97 & 3 \\
Trabalho (seu trabalho ou trabalho do dia a dia) & 82 & 18 \\
Atividades recreativas ou de esportes & &
\end{tabular}


Tabela 2. Porcentagem de compreensão dos itens da nova medida final em português.

\begin{tabular}{lcc}
\hline \multicolumn{1}{c}{ Questão } & $\begin{array}{c}\text { Compreensível } \\
(\mathbf{\%})\end{array}$ & $\begin{array}{c}\text { Não compreensível } \\
\mathbf{( \% )}\end{array}$ \\
\hline Quando você está descansando & 100 & 0 \\
Quando você está realizando uma tarefa com movimentos & 97.5 & 2.5 \\
repetitivos do braço & & \\
Quando você está carregando uma sacola plástica com & 100 & 0 \\
artigos de supermercado & & \\
Qual nota para sua menor dor na semana passada & 97.5 & 2.5 \\
Qual nota para sua maior dor na semana passada & 97.5 & 2.5 \\
Girar uma maçaneta ou chave & 100 & 0 \\
Carregar uma sacola de supermercado ou pasta pelas alças & 100 & 0 \\
Levantar uma xícara de café ou copo de leite até sua boca & 100 & 0 \\
Abrir a tampa de um pote & 100 & 0 \\
Vestir calças & 100 & 0 \\
Torcer um pano de limpeza ou uma toalha molhada & 100 & 0 \\
Atividades pessoais (vestir-se, tomar banho) & 97.5 & 2.5 \\
Trabalhos domésticos (limpeza, manutenção) & 100 & 0 \\
Trabalho (seu trabalho ou trabalho do dia a dia) & 100 & 0 \\
Atividades recreativas/lazer ou de esportes & 100 & 0 \\
\hline
\end{tabular}

Quadro 1. Descritiva para as modificações dos itens não compreensíveis.

\begin{tabular}{|c|c|}
\hline Item do questionário & Modificação para nova versão \\
\hline Quando sua dor foi a menor & Qual nota para sua menor dor na semana passada \\
\hline Quando sua dor foi a pior & Qual nota para sua maior dor na semana passada \\
\hline Atividades recreativas ou de esportes & Atividades recreativas/lazer ou de esportes \\
\hline
\end{tabular}

documentação de mudanças nas condiçôes clínicas do indivíduo. Outro questionário, denominado LES (Liverpool Elbow Score), é mencionado por Nilsson et al. (2008) como utilizado em Liverpool. Contudo, este não focaliza exclusivamente o paciente, contendo dados clínicos.

Em estudo realizado para determinar a sensibilidade do PRFEQ, Newcomer et al. (2005) descrevem que esse questionário é a única ferramenta funcional, de que se tem conhecimento, que é especificamente projetada para o cotovelo e o antebraço.

Segundo Freire, Souza e Lima (2007), as avaliações físicas nem sempre fornecem dados da funcionalidade do cotovelo e das atividades (AVD's) do paciente. Por essa razão, os mesmos enfatizam a necessidade da aplicação de questionário ou escalas visando esse objetivo.

A exclusividade para o cotovelo, seu pequeno tempo de aplicação (NEWCOMER et al., 2005; NILSSON et al., 2008) e mensuração ao longo da semana que antecede seu preenchimento fornecem grande vantagem ao PRTEE (NILSSON et al., 2008). Embora tais vantagens sejam consideradas, se o braço dominante não é o mensurado, o questionário pode se tornar de difícil preenchimento. Outra desvantagem elucidada pelos mesmos autores é o resultado enganoso que a mensuração pode fornecer pela não realização de muitas das tarefas propostas.

Em seus trabalhos, Beaton et al. (2000) e Altan et al. (2010) enfatizam que o aumento do número de projetos de pesquisa - multicultural e multinacional - tem criado a necessidade de adaptar medidas de estado de saúde para outras línguas, além do idioma de origem. Todavia, estes salientam que a adaptação transcultural de um questionário deve se basear em um único método para alcançar equivalência entre a versão fonte e a versão alvo da medida.

Apesar do longo processo exigido para a realização da adaptação cultural, para Guillemin, Bombardier e Beaton (1993) é possível observar várias vantagens na sua realização, dentre as quais: o fornecimento de uma medida comum para a investigaçáo da saúde e da qualidade de vida; uma medida padráo para uso em estudos internacionais, muitos dos quais vêm sendo conduzidos; a permissão de comparação entre grupos nacional/cultural, confiando em uma medida padronizada designada e adaptada para medir o fenômeno transculturalmente; a inclusão 
de imigrantes nas avaliaçóes, evitando o frequente viés de representar somente a cultura dominante do país, e ser um processo menos oneroso e demorado do que o desenvolvimento de uma nova medida.

Para a tradução e a adaptação cultural do PRTEE na língua portuguesa, adotaram-se as normas propostas por Guillemin, Bombardier e Beaton (1993). Nilsson et al. (2008), para adaptar o PRTEE na língua sueca, e Altan et al. (2010), na língua turca, seguiram os critérios propostos por Beaton et al. (2000). Diferentemente dos três estudos citados anteriormente, o trabalho desenvolvido por Leung, Yen e Tse (2004), em Hong Kong, para traduçấo do PRTEE (ainda denominado PRFEQ) na língua chinesa, não faz referência ao método utilizado.

Em relação à equivalência entre a versão de origem e as versōes finais, das línguas traduzidas, foram encontradas semelhanças dos métodos de realização. Neste estudo, no Sueco (NILSSON et al., 2008) e no Turco (ALTAN et al., 2010), a equivalência foi analisada por meio de pré-teste da versão final, enquanto na tradução para o chinês (LEUNG; YEN; TSE, 2004), a equivalência foi mensurada por análise das respostas de 12 pacientes bilíngues.

No processo de tradução e adaptação cultural do PRTEE no Brasil, após aplicação da versão final em português, foram realizadas algumas mudanças para o desenvolvimento da nova versão final em português (Anexo). As modificações realizadas tiveram o objetivo de suprir o nível de não compreensão de algumas questóes e, consequentemente, fornecer o melhor entendimento dos itens reformulados. Os itens "Quando sua dor foi a menor" e "Quando sua dor foi a pior" foram reformulados e modificados para "Qual nota para sua menor dor na semana passada" e "Qual nota para sua maior dor na semana passada" em virtude de a primeira versão do enunciado, que iniciava com a palavra 'quando', não ter sido compreendida adequadamente, uma vez que os participantes relacionavam a dor com situações e locais, em vez de intensidade. Outro item de difícil entendimento referiu-se às "atividades recreativas ou de esportes". Parte dos participantes não conhecia o significado da palavra "recreativa", fator pelo qual a comissão optou por adicionar a palavra "lazer".

Corroborando com a tradução turca, acredita-se que a tradução em português possa também contribuir para estudos científicos de pacientes com epicondilite lateral. As traduçóes permitem comparaçóes transculturais e internacionais (ALTAN et al., 2010). Essas comparaçôes também são citadas por Leung, Yen e Tse (2004), os quais acrescentam que a tradução em chinês também fornece uma simples e uniforme documentação médica para essa doença.

Assim como no presente estudo, os trabalhos realizados por Nilsson et al. (2008) e Altan et al. (2010) obtiveram permissão prévia para sua realizaçáo pelo idealizador do questionário, MacDermid, enquanto a tradução do PRTEE por Leung, Yen e Tse (2004), diferentemente, teve permissão fornecida por Dr Overend, chefe investigador do artigo original que descreve o PRFEQ.

O processo de tradução e adaptação cultural é menos oneroso e trabalhoso do que a criação de uma nova medida de avaliação.

A falta de disponibilidade dos tradutores instituiu dificuldades para formação da comissão.

\section{Conclusão}

Desenvolveu-se uma versão adaptada culturalmente do Patient-rated Tennis Elbow Evaluation (PRTEE) aos profissionais da saúde e pessoas leigas da população brasileira.

Nesta linha de pesquisa, pretende-se incentivar novos estudos para determinar a confiabilidade e a validade do PRTEE na língua portuguesa, bem como a continuidade do estudo por meio da aplicaçáo do questionário a pessoas diagnosticadas com a doença.

\section{Referências}

ALTAN, L.; ERCAN, I.; KONUR, S. Reliability and validity of Turkish version of the patient rated tennis elbow evaluation. Rheumatology International, v. 30 , n. 8, p. 1049-1054, 2010. PMid:19707766. http:// dx.doi.org/10.1007/s00296-009-1101-6

BEATON, D. E.; BOMBARDIER, C.; GUILLEMIN, F.; FERRAZ, M. B. Guidelines for Process of CrossCultural Adaptation of Self-Report Measures. Spine, v. 25, n. 24, p. 3186-3191, 2000. PMid:11124735. http://dx.doi.org/10.1097/00007632-200012150-00014

FREIRE, A. M. F.; SOUZA, R. C.; LIMA, S. M. P. F. Tradução e adaptação cultural do questionário Patient-rated Elbow Evaluation para a lingua portuguesa. 2007. Tese (Especialização em Terapia da Mão e Membro Superior)Universidade Federal de São Paulo, São Paulo, 2007.

FREITAS, P. P. Reabilitação da mão. São Paulo: Atheneu, 2006, p. 351-63.

GUILLEMIN, F.; BOMBARDIER, C.; BEATON, D. Cross-cultural adaptation of health-related quality of life measures: literature review and proposed guidelines. Journal of Clinical Epidemiology, v. 46, n. 12, p. 1417-1432, 1993. http://dx.doi. org/10.1016/0895-4356(93)90142-N

GUILlEMIN, F. Cross-cultural Adaptation and Validation of Health Status Measures. Scandinavian Journal of Rheumatology, v. 24, p. 61-3, 1995. PMid:7747144. http://dx.doi. org/10.3109/03009749509099285

LECH, O.; SEVERO, A. Ombro e cotovelo. In: HERBERT, S.; XAVIER, R.; PARDINI JÚNIOR, A. G.; BARROS FILHOS, T. E. P. Ortopedia e traumatologia - Principios e prática. 3. ed. Porto Alegre: Artmed, 2003. p. 229-32. 
LEUNG, H.; YEN, C.; TSE, P. Reliability of Hong Kong Chinese version of the Patient-rated Forearm Evaluation Questionnaire for lateral epicondylitis. Hong Kong Medical Journal, v. 10, n. 3, p. 172-7, 2004. PMid:15181221.

MACDERMID, J. Update: The Patient-rated Forearm Evaluation Questionnaire is now the Patient-rated Tennis Elbow Evaluation. Journal of Hand Therapy, v. 18, n. 4, p. 407-10, 2005. PMid:16271687. http:// dx.doi.org/10.1197/j.jht.2005.07.002

MACDERMID, J. C. The Patient-rated Tennis Elbow Evaluation (PRTEE)(C) User manual. 2007.

NEWCOMER, K. L.; MARTINEZ-SILVESTRINI J. A.; SCHAEFER, M. P.; GAY, R. E.; ARENDT, K. W. Sensitivity of the Patient-rated Forearm Evaluation Questionnaire in Lateral Epicondylitis. Journal of Hand Therapy, v. 18, p. 400-406, 2005. PMid:16271686. http://dx.doi.org/10.1197/j.jht.2005.07.001

NILSSON, P.; BAIGI, A.; MARKLUND, B.; MÅNSSON, J. Cross-cultural adaptation and determination of the reliability and validity of PRTEE-S (Patientskattad Utvärdering av Tennisarmbåge), a questionnaire for patients with lateral epicondylalgia , in a Swedish population. BMC Musculoskeletal Disorders, v. 9, p. 79, 2008. PMid:18534009. PMCid:2435532. http:// dx.doi.org/10.1186/1471-2474-9-79

PARDINI JÚNIOR, A. G. Lesôes por esforços repetitivos. In: PARDINI JÚNIOR, A. G. Traumatismos da mão. 3. ed. MEDSI, 2000. p. 163.

PARDINI JUNIOR, A. G.; FREITAS, A. D.; TAVARES, K. E. Antebraço, punho e mão. In: HERBERT, S.; XAVIER, R.; PARDINI JUNIOR, A. G.; BARROS FILHO, T. E. P. Ortopedia e traumatologia - Princípios e prática. 3. ed. Porto Alegre: Artmed, 2003. p. 265.

ROMPE, J. D.; OVEREND, T. J.; MACDERMID, J. C. Validation of the Patient-rated Tennis Elbow Evaluation Questionnaire. Journal of Hand Therapy, v. 20, n.1, p. 3-11, 2007. PMid:17254903. http:// dx.doi.org/10.1197/j.jht.2006.10.003

\section{Autores}

\section{Caroline Sousa de Andrade}

Terapeuta ocupacional, Especialista em Terapia da Mão pela UNIFESP-EPM, Professora do curso de Terapia ocupacional da Universidade Potiguar - UNP, Natal, RN, Brasil

\section{Regyane Costa Souza}

Terapeuta ocupacional, Especialista em Terapia da Mão pela Faculdade de Medicina da Universidade de São Paulo - FMUSP, Coordenadora da Pós-Graduação lato sensu em Terapia da Mão e Membro Superior da UNIFESP-EPM, São Paulo, SP, Brasil

\section{Therezinha Rosane Chamlian}

Fisiatra, Doutora, Professora afiliada do Departamento de Ortopedia e Traumatologia da UNIFESP/SP, Diretora técnica do Centro de Reabilitação Lar Escola São Francisco, São Paulo, SP, Brasil

\section{Marcelo Hide Matsumoto}

Médico, Doutor e Chefe do Grupo do Ombro e Cotovelo da UNIFESP-EPM, São Paulo, SP, Brasil

\section{Joáo Baptista Gomes dos Santos}

Médico, Doutor, Professor Adjunto e Chefe do Departamento de Ortopedia e Traumatologia, Chefe da disciplina de Cirurgia da Mão e Membro Superior da Universidade Federal de São Paulo - UNIFESP-EPM, São Paulo, SP, Brasil

\section{Akira Ishida}

Médico, Doutor e Membro titular do Departamento de Ortopedia e Traumatologia da Universidade Federal de São Paulo - UNIFESP-EPM, São Paulo, SP, Brasil

\section{Contribuição dos Autores}

Regyane: concepção do manuscrito, pesquisa bibliográfica e revisão. Caroline: pesquisa bibliográfica, redação do texto e revisão. Os demais são revisores.

\section{Notas}

${ }^{1}$ Estudo desenvolvido e apresentado em forma de trabalho de conclusão de curso para aquisição de título de Especialização em Terapia da Mão e Membro Superior, na Universidade Federal de São Paulo (UNIFESP/EPM), desenvolvido no Departamento de Ortopedia e Traumatologia da UNIFESP. Parte dos dados foi apresentada no VIII Congresso NorteNordeste de Terapia Ocupacional. O estudo foi apresentado no XI Congresso Brasileiro de Reabilitação da Mão e o resumo desse artigo publicado em anais do mesmo. 